\title{
Hand-biting Lesions in a Child - a Challenging Diagnosis
}

\author{
Anca Chiriac ${ }^{1,2,3}$, Cristian Podoleanu4 ${ }^{4}$ Simona Stolnicu, 5,6 \\ 1 Nicolina Medical Center, Department of Dermatology, Iași, Romania \\ 2 Apollonia University, Iași, Romania \\ 3 P. Poni Research Institute, Romanian Academy, Iași, Romania \\ 4 Department of Internal Medicine, University of Medicine and Pharmacy, Tîrgu Mureș, Romania \\ 5 Department of Pathology, University of Medicine and Pharmacy, Tîrgu Mureș, Romania \\ ${ }^{6}$ Histopat Invest Laboratory, Tîrgu Mureş, Romania
}

\section{CORRESPONDENCE}

\section{Cristian Podoleanu}

Str. Gheorghe Marinescu nr. 38

540139 Tîrgu Mureş, Romania

Tel: +40 265215551

E-mail: podoleanu@me.com

\section{ARTICLE HISTORY}

Received: October 20, 2017

Accepted: December 15, 2017
Anca Chiriac • Str. Hatman Sendrea nr. 2, 700613 lași, Romania. Tel: +40 332808703

Simona Stolnicu • Str. Gheorghe Marinescu nr. 38 540139 Tîrgu Mures, Romania. Tel: +40 265215551

\section{ABSTRACT}

Background: Self-induced skin lesions, especially in young children, can create confusion within pediatricians, dermatologists, or other medical care providers, leading to different diagnoses, unnecessary investigations, and delaying the correct therapeutic psychiatric evaluation. Case report: We report the case of a 4-year-old boy who was referred to Dermatology after being hospitalized in the Allergy Department for a chronic allergic contact dermatitis. He had been previously diagnosed with chronic hand dermatitis, atopic dermatitis, and psoriasis, and treated with no favorable outcome. Scaly erythematous plaques were noticed on the dorsal aspects of both hands and on the lateral folds of the fingers. The skin lesions were distributed in a nonsymmetrical way. A diagnosis of self-injurious behavior was presumed, and psychiatric evaluation was asked. The child was transferred to the Psychiatry Department, and a diagnosis of schizophrenia was concluded.

Keywords: allergic contact dermatitis, risk assessment, treatment

\section{INTRODUCTION}

Self-induced skin lesions, especially in young children, can create confusion within pediatricians, dermatologists, or other medical care providers, leading to different diagnoses, unnecessary investigations, and delaying the correct therapeutic psychiatric evaluation. The mainstay of the diagnosis is to think about it.

\section{CASE REPORT}

A 4-year-old boy was referred to Dermatology after being hospitalized several days in the Allergy Department for a chronic allergic contact dermatitis. He had been previously diagnosed with chronic hand dermatitis, atopic dermatitis, and 


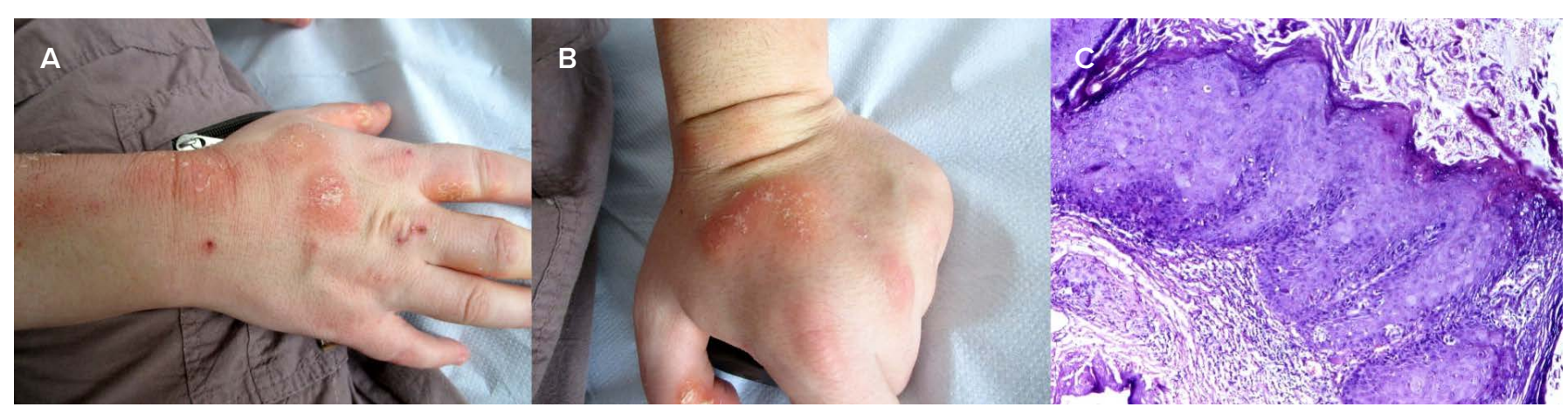

FIGURE 1. Eczematous, excoriated, crusted plaques on the right (A) and left (B) hand. Hyperkeratosis, acanthosis, and mild inflammatory infiltrate in the superficial dermis (Hematoxylin-Eosin, 10x) (C).

psoriasis, and treated with oral antihistamines, topical steroids, and emollients, with no favorable outcome.

The dermatological examination discovered non-symmetrical scaly erythematous plaques on the dorsal aspects of both hands and on the lateral folds of the fingers (Figure 1A, 1B). Pruritus, pain, or any other complains were denied by the mother. During clinical examination, the child seemed relaxed, non-communicative at the beginning, but with compulsive acts of self-biting and sucking of the hands. A detailed family history search for dermatological and/or psychiatric disorders was undertaken and ruled out any medical issues.

Exhaustive laboratory investigations were carried out, including patch tests, prick tests, and direct mycological examinations, and were all negative. A punch biopsy revealed hyperkeratosis, acanthosis, and mild inflammatory infiltrate in the superficial dermis (Figure 1C).

A diagnosis of self-injurious behavior was presumed, and psychiatric evaluation was asked. The child was transferred to the Psychiatry Department, and a diagnosis of schizophrenia was concluded, which required psychiatric hospitalization. The skin lesions were treated with emollients, and the patient received psychiatric treatment, with a good response to treatment as assessed during follow-up.

The Declaration of Helsinki protocols were followed during our study and the patient's family gave their written, informed consent.

\section{DISCUSSIONS}

Self-injurious behavior is a syndrome characterized by self-induced physical damage such as nail and finger biting, head banging, oral and genital mutilation. ${ }^{1}$

Dermatitis artefacta covers a large spectrum of self-inflicted but involuntarily induced skin lesions, unlike neurotic excoriations, Munchausen syndrome, and true malingering. ${ }^{2}$

Self-mutilation could be considered as a "cry for attention” or a behavior that reduces anxiety, coexisting with psychiatric disorders; $66.12 \%$ of persons with severe selfinduced skin lesions were diagnosed with schizophrenia. ${ }^{3}$

Usually, such patients, regardless of their age, are seen first by dermatologists, without psychiatric examination, inducing an important delay in a correct diagnosis and treatment. Moreover, antipsychotic treatment improves cutaneous lesions along with the psychiatric illness. ${ }^{4}$

There is a close correlation between schizophrenia and dermatitis. A recently published study indicates an association between atopic disorders and schizophrenia. ${ }^{5}$ Contact dermatitis and other types of eczema have been found in a higher incidence in patients diagnosed previously with schizophrenia. ${ }^{6}$

\section{CONCLUSIONS}

Although the diagnosis of self-induced lesions in young children is a challenge for pediatric physicians and dermatologists, a high awareness of a psychiatric disorder must be maintained.

\section{CONFLICT OF INTEREST}

None for all authors.

\section{REFERENCES}

1. Ghanizadeh A. Nail biting; etiology, consequences and management. Iran J Med Sci. 2011;36:73-79.

2. Bergler-Czop B, Brzezińska-Wcisło L. Psychodermatologic disorders: case reports and review of literature. Postep Derm Alergol. 2012;29:401-406.

3. Millard LG, Cotterill JA. Psychocutaneous disorders. In Rook's textbook of dermatology (eds Burns T, Breathnach S, Cox N): 1-5. Blackwell Science, 2004.

4. Schennach R, Riedel M, Musil R, et al. Treatment Response in First-episode Schizophrenia. Clin Psychopharmacol Neurosci. 2012;10:78-87.

5. Pedersen MS, Benros ME, Agerbo E, et al. Schizophrenia in patients with atopic disorders with particular emphasis on asthma: a Danish populationbased study. Schizophr Res. 2012;138:58-62.

6. Weber NS, Cowan DN, Millikan AM, et al. Psychiatric and general medical conditions comorbid with schizophrenia in the National Hospital Discharge Survey. Psychiatr Serv. 2009;60:1059-1067. 